\title{
Ground red hot pepper agar in the isolation of yeasts of Candida spp.
}

\author{
S. STEPANOVIĆ, S. DJUKIĆ, D. VUKOVIĆ, S. MITROVIĆ* and D. BABIĆ $\dagger$ \\ Departments of Bacteriology and *Parasitology, Institute of Microbiology and Immunology and $\dagger$ Institute for \\ Statistics and Informatics, School of Medicine, University of Belgrade, Belgrade, Yugoslavia
}

\begin{abstract}
The purpose of this study was to investigate and determine the value of a novel, simple and inexpensive selective medium for isolation of yeasts of Candida spp. - ground red hot pepper agar (GRHP). The study compared GRHP and Sabouraud dextrose agar (SDA), an insufficiently selective medium routinely used for primary isolation of yeasts. The comparison was based on qualitative and quantitative characterisation of growth of 25 bacterial strains, measurement of growth of 22 yeast strains and testing on clinical specimens. Qualitative tests on bacteria showed either significantly less growth on GRHP than on SDA, or no growth on GRHP. Quantitative tests confirmed these results; the number of colonies of all tested bacterial species and strains on GRHP was significantly lower than on SDA. With regard to the isolation of Candida spp., GRHP had the same properties as SDA. Statistical analysis showed no significant differences in the growth of Candida spp. and strains on the two media. All these results were confirmed by tests on clinical material. The results clearly show that GRHP agar is an economical medium for the isolation of yeasts of Candida spp., with excellent selectivity.
\end{abstract}

\section{Introduction}

Yeasts, especially Candida spp., are very important human pathogens [1]. Yeasts have emerged as a highly significant cause of opportunistic infections, particularly in immunocompromised patients [2-4], and also play an important role as proven pathogens in nosocomial infections $[5,6]$. As for other microorganisms, there is always a need for reliable, easy to prepare and inexpensive medium for primary isolation. Various media are available for the primary isolation of yeasts, but undoubtedly the most popular is Sabouraud dextrose agar. There is no special need for another medium when the sample is free of bacteria. However, when the specimen is taken from a contaminated site (e.g., throat, bowel or vagina), the isolation of yeasts can be difficult and time consuming. In such cases a selective medium that suppresses the contaminating flora is needed. Several types of selective media have been used for primary isolation of yeasts, e.g., Sabouraud-gentamicin agar, oxytetracycline glucose yeast agar, Nickerson agar and CHROMagar [7-10]. In a search for a simple, inexpensive and effective medium for the culture of yeasts, 15 readily available

Received 3 Sept. 1997; revised version accepted 2 March 1998.

Corresponding author: Dr S. Stepanović. plant extracts were tested in this laboratory for their use as selective agents. Preliminary experiments showed ground red hot pepper to be potentially useful. The objective of the present study was to investigate and determine the value of this novel, simple and inexpensive selective medium for the isolation of yeasts of Candida spp.

\section{Materials and methods}

\section{Media}

Columbia agar (CA; Torlak, Yugoslavia) with sheep blood 5\%, Sabouraud Dextrose Agar (SDA) with dextrose (Torlak) 4\% and ground red hot pepper agar (GRHP) were used in this study. CA and SDA were prepared according to the manufacturer's instructions. The GRHP was made by mixing ground red hot pepper (D.D. Aleva, Novi Knezevac, Yugoslavia) 20 g, dextrose (Torlak) $2 \mathrm{~g}$ and agar (Bacto Agar, Difco) $7.5 \mathrm{~g}$ in $500 \mathrm{ml}$ of distilled water. This mixture was heated to boiling point and then sterilised in an autoclave for $30 \mathrm{~min}$ at $116^{\circ} \mathrm{C}$. After sterilisation and slight cooling, GRHP was mixed vigorously, to avoid segregation of the red hot pepper and then poured into plastic petri dishes. The final $\mathrm{pH}$ was 4.9 (pH-meter IQ 200, IQ Scientific Instruments). After the medium had solidi- 
fied, plates were inverted and allowed to dry overnight at room temperature.

\section{Micro-organisms}

A selected set of 25 bacterial strains and 22 yeasts was used. The following gram-negative fermentative bacteria were used: Salmonella enteritidis, Shigella sonnei, Citrobacter freundii, Escherichia coli ATCC 25922, E. coli ATCC 35218, Serratia marcescens, Proteus mirabilis, Providencia stuartii, Morganella morganii, Enterobacter sp. and Yersinia enterocolitica. Gramnegative non-fermentative bacteria used in the study were: Pseudomonas aeruginosa, Acinetobacter sp., Comamonas acidovorans, Xanthomonas maltophilia and Sphingobacterium multivorum. Gram-positive bacteria studied were: Staphylococcus aureus ATCC 25923, Enterococcus faecalis ATCC 29212, Micrococcus sp., Staph. aureus, Staph. epidermidis, Listeria monocytogenes CIP 12505 (Collection de l'Institut Pasteur), Bacillus cereus CIP 5832, $\beta$-haemolytic streptococcus group B, and Enterococcus sp. The type strains were obtained from the American Type Culture Collection and Collection de l'Institut Pasteur. With the exception of the type collection strains, all the bacteria were clinical isolates, isolated and identified in this laboratory by currently accepted identification criteria [11]. After transferring the inocula from the stock culture to $\mathrm{CA}$, all the bacterial strains were incubated at $37^{\circ} \mathrm{C}$ for $24 \mathrm{~h}$ in air. Yeasts used for the study were: Candida albicans (12 strains), C. parapsilosis (3), $C$. tropicalis (3), C. lusitaniae, C. krusei, C. guilliermondii and $C$. glabrata. All the yeasts were also clinical isolates, identified by API20 C AUX (bioMérieux, France). Following the transfer of inocula to SDA, all the yeasts were incubated at $37^{\circ} \mathrm{C}$ for $24 \mathrm{~h}$ in air. To avoid bias in reading the results, all the strains were referred to by code numbers.

\section{Experimental design}

Qualitative test for bacteria. For each bacterial strain, five or six single colonies were picked with a bacteriological loop and suspended in $1 \mathrm{ml}$ of sterile distilled water (SDW); $100 \mu$ l of each suspension were transferred to each of CA, SDA and GRHP with an automatic pipette and spread over the surface of the whole plate with a glass rod. The plates were observed after incubation at $37^{\circ} \mathrm{C}$ for $48 \mathrm{~h}$ in air.

Quantitative test for bacteria. Quantitative tests were performed only for bacterial strains that showed visible growth in the qualitative test. The bacteria were harvested after incubation for $24 \mathrm{~h}$ at $37^{\circ} \mathrm{C}$ on $\mathrm{CA}$, and suspended in $3 \mathrm{ml}$ of SDW to obtain c. $10^{7} \mathrm{cfu} / \mathrm{ml}$. The suspension was then diluted in 10-fold steps to a dilution of $10^{-4}$ in SDW. Samples $(100 \mu \mathrm{l})$ from the resulting $10^{3} \mathrm{cfu} / \mathrm{ml}$ dilution were spread over the surface of CA, SDA and GRHP as above. After incubation at $37^{\circ} \mathrm{C}$ for $72 \mathrm{~h}$ in air, colonies were counted.

Quantitative test for yeasts. Yeast strains were harvested after incubation for $24 \mathrm{~h}$ at $37^{\circ} \mathrm{C}$ on SDA and cells were suspended in $3 \mathrm{ml}$ of SDW to obtain $10^{6} \mathrm{cfu} / \mathrm{ml}$. The number of cells was checked with a Neubauer's counting chamber (haemocytometer). The suspension was subjected to three 10 -fold dilutions in SDW, and 100- $\mu$ l samples from the resulting $10^{3} \mathrm{cfu} / \mathrm{ml}$ dilution were spread over SDA and GRHP as above. After incubation at $37^{\circ} \mathrm{C}$ for $72 \mathrm{~h}$ in air, colonies were counted.

Testing of GRHP on clinical specimens. Two vaginal swabs were taken from each of 103 out-patients attending an infertility clinic at the Institute of Microbiology and Immunology. One was inoculated on SDA and the other on GRHP, in a random order. After incubation at $37^{\circ} \mathrm{C}$ for $48 \mathrm{~h}$ in air, plates were visually inspected for the presence of yeasts, which were confirmed by microscopic examination.

Statistical analysis. The statistical significance of differences in numbers of colonies (cfu) of various bacteria on CA, SDA, and GRHP was tested by Fisher's one-way analysis of variance (ANOVA) for proportion followed by Fisher's least significant difference test. To evaluate differences in yeast growth on SDA and GRHP the Pearson's $\chi^{2}$ goodness of fit test was used. The level of statistical significance used was $\mathrm{p}<0.05$.

\section{Results}

\section{Qualitative test for bacteria}

All organisms showed visible growth on CA, which served as the control medium. On SDA, the growth of all bacteria was also visible, but not as pronounced as on CA. However, bacteria growing on GRHP showed fewer and smaller colonies (S. enteritidis, Cit. freundii, $E$. coli ATCC 25922, E. coli ATCC 35218, Ser. marcescens, Enterobacter sp., Acinetobacter sp., Ent. faecalis ATCC 29212, Enterococcus sp.) or an absence of growth.

\section{Quantitative test for bacteria}

In this test, the growth of bacteria that showed visible growth in the previous experiment was determined quantitatively. As a significantly lower number of bacteria was used in this test, a more precise observation of growth dynamics and appearance of colonies was possible. Enterobacter sp. was the only bacterium whose colonies could be reliably counted (diameter c. 2-2.5 mm) after incubation for only $48 \mathrm{~h}$ on GRHP. Acinetobacter sp., Staph. aureus and Staph. epidermidis did not show visible growth even after incubation for 7 days. All the other bacteria showed no 
visible colonies after incubation for $24 \mathrm{~h}$, while after $48 \mathrm{~h}$ very small, pin-point colonies could be seen. Finally, after incubation for $72 \mathrm{~h}$, small colonies of $c$. $1-1.5 \mathrm{~mm}$ in diameter were counted. Table 1 shows the numbers of counted colonies of the tested bacteria on CA, SDA and GRHP. Compared with CA as a control medium, the number of colonies of $S$. enteritidis, Ser. marcescens, Ent. faecalis ATCC 29212, Staph. aureus and Staph. epidermidis on SDA was significantly lower $(p<0.05)$, while the growth of other tested bacteria showed no difference $(p>0.05)$. However, the number of colonies of all tested species and strains on GRHP was significantly lower compared with those grown on both CA $(p<0.01)$ and SDA $(p<0.01)$.

\section{Quantitative test for yeasts}

The growth of 22 species and strains of Candida spp. on SDA and GRHP was quantitatively determined. After incubation for $24 \mathrm{~h}$ at $37^{\circ} \mathrm{C}$ in air, colonies of all yeasts were clearly visible on the plates. Nevertheless, they were incubated for a further $24 \mathrm{~h}$ and then counted, because after prolonged incubation colonies were larger and easier to count. The appearance of colonies (shape, size, colour, consistency) on SDA and GRHP was similar. Statistical analysis showed no difference in quantity of growth on SDA (62-185 colonies) and GRHP (55-172) for all yeast strains tested $(\mathrm{p}>0.05)$.

\section{Testing of GRHP with clinical specimens}

The results of the comparison of SDA and GRHP for the isolation of yeasts from clinical specimens are shown in Table 2. Of the total of 103 vaginal samples, 19 were positive for yeasts: 17 on SDA, and 17 on GRHP. Growth was observed on both SDA and GRHP in 15 cases, and the number of colonies observed was similar on the two media. Growth on SDA only (one

Table 1. Quantitative characterisation of growth of bacteria on Columbia blood agar (CA), Sabouraud dextrose agar (SDA) and ground red hot pepper agar (GRHP)

\begin{tabular}{lrrr}
\hline & \multicolumn{3}{c}{ cfu on } \\
\cline { 2 - 4 } Organism & CA & SDA & GRHP \\
\hline Salmonella enteritidis & 169 & $117^{*}$ & $3^{\dagger}$ \\
Citrobacter freundii & 193 & 187 & $1^{\dagger}$ \\
E. coli ATTC 25922 & 59 & 62 & $20^{\dagger}$ \\
E. coli ATTC 35218 & 231 & 210 & $55^{\dagger}$ \\
Ser. marcescens & 74 & $58^{*}$ & $2^{\dagger}$ \\
Enterobacter sp. & 153 & 144 & $20^{\dagger}$ \\
Acinetobacter sp. & 150 & 137 & $0^{\dagger}$ \\
Ent. faecalis ATCC 29212 & 433 & $316^{*}$ & $132^{\dagger}$ \\
Staph. aureus & 199 & $27^{*}$ & $0^{\dagger}$ \\
Staph. epidermidis & 590 & $293^{*}$ & $0^{\dagger}$ \\
Enterococcus sp. & 592 & 568 & $99^{\dagger}$ \\
\hline
\end{tabular}

\footnotetext{
${ }^{*}$ Statistically significant compared with CA $(\mathrm{p}<0.05)$.

'Statistically significant compared with both $\mathrm{CA}$ and SDA $(\mathrm{p}<0.01)$.
}

Table 2. Comparison of SDA and GRHP for the isolation of yeasts from 103 clinical samples

\begin{tabular}{lcc}
\hline & \multicolumn{2}{c}{ Number of colonies on } \\
\cline { 2 - 3 } Specimen no. & SDA & GRHP \\
\hline 1 & 1 & 0 \\
2 & +++ & +++ \\
3 & 39 & 15 \\
4 & 1 & 0 \\
5 & +++ & +++ \\
6 & +++ & ++ \\
7 & 4 & 3 \\
8 & +++ & +++ \\
9 & +++ & +++ \\
10 & 0 & 1 \\
11 & +++ & +++ \\
12 & 12 & 3 \\
13 & +++ & +++ \\
14 & 0 & 1 \\
15 & +++ & +++ \\
16 & +++ & +++ \\
17 & ++ & ++ \\
18 & 1 & 4 \\
19 & +++ & +++ \\
\hline
\end{tabular}

,$+++>200$ colonies;,$++ 100-200$ colonies.

colony) was observed in two cases, as was growth on GRHP only (one colony).

\section{Discussion}

The main deficiency of SDA as a medium for the routine primary isolation of yeasts is its insufficient selectivity. Various additives improve selectivity, but these increase the price and complicate the preparation procedure. In the present study, an alternative medium for the isolation of yeasts of Candida spp. was designed and tested. The results showed that ground red hot pepper medium has excellent selectivity. Qualitative tests on bacteria showed significantly less growth, or the complete lack of it, on GRHP as compared with SDA. Most of the bacterial strains that did grow on GRHP produced very small pin-point colonies, which did not interfere with the isolation of yeasts as the latter were easily distinguishable. Quantitative analyses confirmed these results. The substantial reduction in growth of all the bacteria on GRHP, compared with both CA and SDA, has high statistical significance $(p<0.01)$. With regard to the isolation of Candida spp., the GRHP has the same properties as the SDA; quantitative analyses showed no statistically significant differences $(p>0.05)$ in growth of Candida spp. and strains on these two media. These results were confirmed by tests on clinical specimens. The few minor discrepancies between SDA and GRHP in the isolation of yeasts from clinical samples were probably due to the use of two separate vaginal swabs, containing different numbers of organisms.

The ground red hot pepper used in the medium is a spice, used for human consumption. The production of this spice in Yugoslavia is regulated by the Yugosla- 
vian federal law [12], so that its contents and quality are constant. The GRHP medium is not absolutely standardised, but that is also the case with SDA. The type of peptone used in Sabouraud medium, as well as the type of agar and the conditions of autoclave sterilisation, influence the quality of this medium [13].

The mechanism by which the GRHP medium inhibits the growth of bacteria is not known. There are two obvious candidates for the inhibiting mechanism: low nutritious value of GRHP for bacteria or low $\mathrm{pH}$ value of the medium. However, the possibility cannot be discounted that some other, less transparent mechanism is responsible for the inhibition of bacterial growth on GRHP medium. It should be carefully noted that the GRHP is subjected to high temperatures during the autoclaving step, which may degrade or destroy some of its chemical components. For example, one component of the medium is capsaicin. Capsaicin, a homovanillic acid derivative (8-methyl-Nvanillyl-6-nonenamide) is the major pungent ingredient of plants of the genus Capsicum such as hot green and red peppers [14]. It is possible that capsaicin has a negative selective influence on the growth of prokaryotic bacteria, but not on eukaryotic yeasts. Some recent results indicate that capsaicin has a bactericidal effect for some bacteria [15]. According to Yugoslav standards, ground red hot pepper must contain $0.05-0.07 \%$ capsaicin, whereas sweet ground red pepper should contain capsaicin only in traces. We have performed the qualitative test for bacteria with sweet ground red pepper and have observed no significant differences in growth response of bacteria on the two media. However, the qualitative nature of these tests does not allow a complete exclusion of capsaicin as a potential inhibitor.

While the mechanism of selectivity of GRHP remains unknown, these results clearly show that it is an excellent selective and economical medium for the isolation of Candida spp. Further experiments are currently underway to identify the mechanism of selectivity of GRHP and to test the possible applica- tion of GRHP as a medium for the isolation of other yeasts and moulds.

This study was supported in part by a grant from the Ministry of Science and Technology of the Republic of Serbia.

\section{References}

1. Odds FC. Candida infections: an overview. Crit Rev Microbiol 1987; 15: $1-5$

2. Saral R. Candida and Aspergillus infections in immunocompromised patients: an overview. Rev Infect Dis 1991; 13: 487-492.

3. Wingard JR. Infections due to resistant Candida species in patients with cancer who are receiving chemotherapy. Clin Infect Dis 1994; 19 Suppl 1: S49-S53.

4. Nieto-Rodriguez JA, Kusne S, Manez $\mathrm{R}$ et al. Factors associated with the development of candidemia and candidemia-related death among liver transplant recipients. Ann Surg 1996; 223: 70-76.

5. Jarvis WR. Epidemiology of nosocomial fungal infections, with emphasis on Candida species. Clin Infect Dis 1995; 20: 1526- 1530 .

6. Fridkin SK, Jarvis WR. Epidemiology of nosocomial fungal infections. Clin Microbiol Rev 1996; 9; 499-511.

7. Merz WG, Sandford G, Evans GL. Clinical evaluation of the addition of gentamicin to commercially prepared mycological media. J Clin Microbiol 1976; 3: 496-500.

8. Mossel DAA, Kleynen-Semmeling AMC, Vincentie HM, Beerens H, Catsaras M. Oxytetracycline-glucose-yeast extract agar for selective enumeration of moulds and yeasts in foods and clinical material. J Appl Bacteriol 1970; 33: 454- 457 .

9. Bergman JJ, Berg AO, Schneeweiss R, Heidrich FE. Clinical comparison of microscopic and culture techniques in the diagnosis of Candida vaginitis. $J$ Fam Pract 1984; 18: $549-552$.

10. Bernal S, Martin Mazuelos E, Garcia M. Aller AI, Martinez MA, Gutierrez MJ. Evaluation of CHROMagar Candida medium for the isolation and presumptive identification of species of Candida of clinical importance. Diagn Microbiol Infect Dis 1996; 24: 201-204.

11. Murray PR, Baron EJ, Pfaller MA, Tenover FC, Yolken RH (eds) Manual of clinical microbiology, 6th edn. Washington, DC, ASM Press. 1995

12. Yugoslav Official Register 1979; 1 (January 5th): 21

13. Odds FC. Sabouraud('s) agar. J Med Vet Mycol 1991; 29: 355-359.

14. Cordell GA, Araujo OE. Capsaicin: identification, nomenclature, and pharmacotherapy. Ann Pharmacother 1993; 27: $330-336$.

15. Jones NL, Shabib S, Sherman PM. Capsaicin as an inhibitor of the growth of the gastric pathogen Helicobacter pylori. FEMS Microbiol Lett 1997; 146: 223-227. 